\title{
Tapinarof Cream 1\% Once Daily for Plaque Psoriasis: Long-Term Extension Trial of a Novel Therapeutic Aryl Hydrocarbon Receptor Modulating Agent
}

Bruce Strober, ${ }^{1}$ Linda Stein Gold 2 Robert Bissonnette ${ }^{3}$ April Armstrong, ${ }^{4}$ Andrew Blauvelt ${ }^{5}$ Leon Kircik 6,7 Philip M. Brown, ${ }^{8}$ Anna M. Tallman, ${ }^{8}$ Mark Lebwohl

\begin{abstract}
Yale University, New Haven \& Central Connecticut Dermatology Research, Cromwell, CT, USA, ${ }^{2 H e n r y}$ Ford Health System, Detroit, MI, USA, Innnovaderm Research Inc., Montreal, QC, Canada, "KKeck School of Medicine at University of Southern California, Los Angeles,
\end{abstract}

\begin{tabular}{l}
\hline SYNOPSIS \\
In two 12-week pivotal phase 3 trials, PSOARING 1 (NCT03956355) and \\
PSOARING 2 (NCT03983980), tapinarof cream $1 \%$ once daily (QD) demonstrated \\
highly statistically and clinically significant efficacy versus vehicle and was well \\
tolerated in adults with mild to severe plaque psoriasis 1 \\
Tapinarof cream $1 \%$ QD also demonstrated maintenance of efficacy for 4 weeks \\
after treatment discontinuation in a 12-week phase $2 \mathrm{~b}$ trial, warranting further \\
investigation of a potential remittive effect ${ }^{2}$ \\
\hline OBJECTIVE
\end{tabular}

To present the results of PSOARING 3 (NCT04053387), a long-term extension trial designed to assess the safety, efficacy, durability of response, tolerability, and duration of remittive effect of tapinarof during repeated interm
based on patient Physician Global Assessment (PGA) score

\section{METHODS}

\section{Study Design}

Patients completing PSOARING 1 and PSOARING 2 were eligible to enroll in

PSOARING 3 for 40 weeks of open-label treatment with tapinarof cream $1 \%$ OD,

followed by four weeks of follow-up (Figure 1 )

In PSOARING 3, patients were treated with tapinarof 1\% QD based on individual

- Patients who entered with a PGA score of $\geq 1$ received tapinarof $1 \%$ OD until complete disease clearance was achieved, defined as a PGA score of $O$ Patients who entered with, or achieved, a PGA score of 0 discontinued treatment and were observed for remittive effect, defined as maintenance of

If disease worsening occurred, defined as a PGA score $\geq 2$, tapinarof $1 \% \mathrm{OD}$ was started and continued until a PGA score of 0 (clear) was achieved

Figure 1. PSOARING 3 Study Design

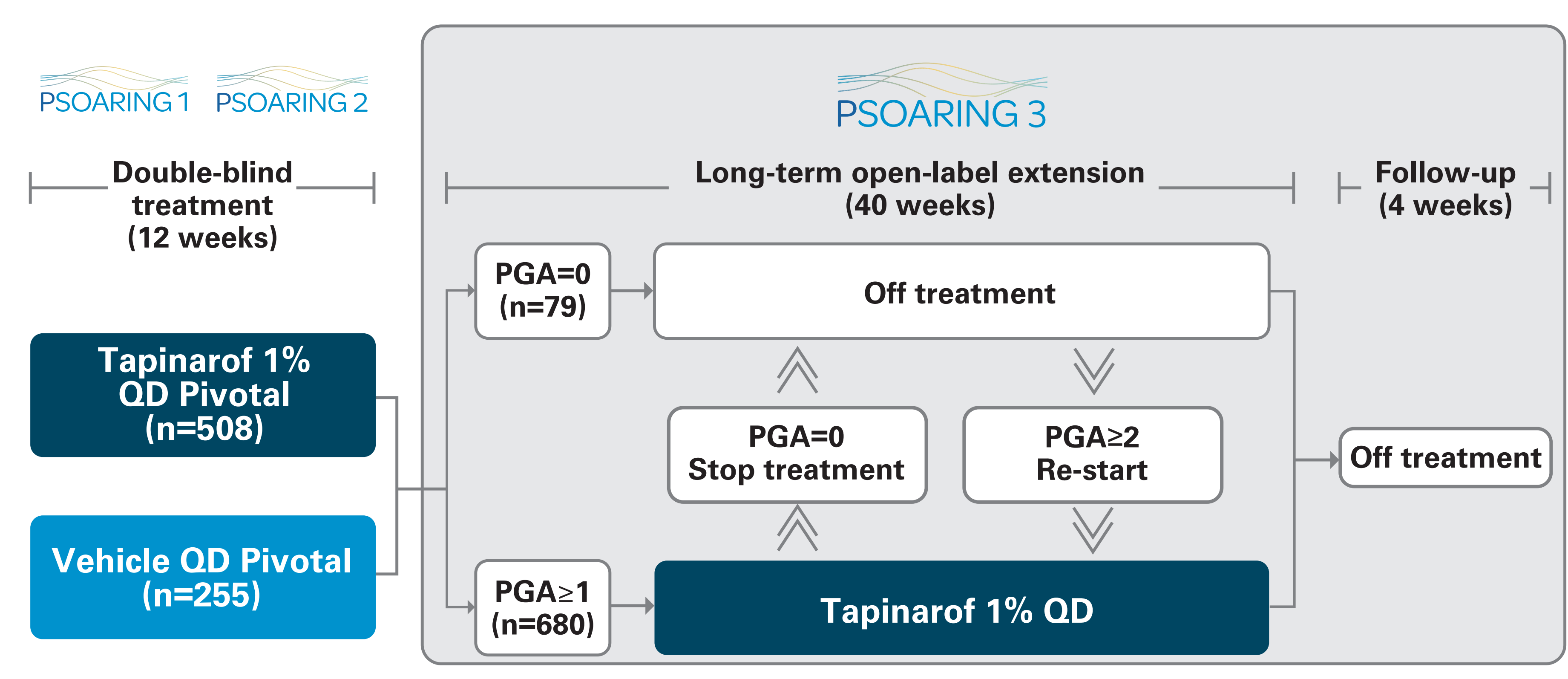

Endpoints and Statistical Analysis

Safety: Adverse events (AEs), laboratory values, vital signs and physical exams

Efficacy:

Complete Disease Clearance: Proportion of patients achieving PGA of 0 (clear) - Remittive Effect: Duration of efficacy maintenance defined as PGA of 0 (clear) or 1 (almost clear) while off therapy after achieving complete disease clearance

Response: Proportion of patients who entered the trial with a $P G A \geq 2$ and achieved a PGA of 0 (clear) or 1 (almost clear) at least once during the trial - Durability of Response (absence of tachyphylaxis): Maintenance of efficacy while on treatment, defined as the proportion of patients who achieved a PGA score Index (PASI) score and percentage of body surface area (\%BSA) affected Index (PASI)

- Tolerability: Local tolerability using a patient-reported 5 -point scale for burning/ stinging and itching, and an investigator-
for dryness, erythema, and peeling

- Efficacy analyses used observed case (OC) or last observation carried forward analysis that were based on the intention-to-treat (ITT) population

\section{RESULTS}

Baseline Patient Demographics and Disease Characteristics

Overall, 763 (91.6\%) of eligible patients completing PSOARING 1 and

PSOARING 2 opted to enroll in PSOARING 3

Patient demographics and disease characteristics are summarized in Table 1 Pcluding baseline values by prior treatment arm in the pivotal trials

Patients previously randomized to tapinarof 1\% QD (Tapinarof $\rightarrow$ Tapinarof) had lower baseline disease scores compared to the vehicle $Q D$ (Vehicle $\rightarrow$ Tapin
group, reflecting the significant efficacy of tapinarof in the pivotal studies $-14.6 \%(74 / 508)$ versus $2.0 \%(5 / 255)$ of patients had complete disease clearance of 1 (almost clear) or 2 (mild) in the tapinarof OD pivotal group (Tapinarof $\rightarrow$ Tapinarof) versus the vehicle OD pivotal group, (Vehicle $\rightarrow$ Tapinarof) respectively
Table 1. PSOARING 3 Baseline Patient Demographics and Disease Characteristics

\begin{tabular}{|c|c|c|c|}
\hline & Overall $(n=763)$ & $\begin{array}{c}\text { Tapinarof } \rightarrow \\
\text { Tapinarof* } \\
(n=508)\end{array}$ & $\begin{array}{c}\text { Vehicle } \rightarrow \\
\text { Tapinarof* } \\
\text { (n=255) }\end{array}$ \\
\hline Age, years, mean (SD) & $50.7(12.88)$ & 50.5 (12.87) & $51.0(12.93)$ \\
\hline Male, n (\%) & $448(58.7)$ & $304(59.8)$ & $144(56.5)$ \\
\hline Weight, kg, mean (SD) & $92.4(23.90)$ & 92.6 (25.13) & $92.1(21.28)$ \\
\hline $\mathrm{BMI}, \mathrm{kg} / \mathrm{m}^{2}$, mean (SD) & $31.7(7.71)$ & $31.6(8.07)$ & $31.8(6.97)$ \\
\hline
\end{tabular}

PGA, $\mathbf{n}(\%)^{+}$

\begin{tabular}{|l|c|c|c|}
\hline 0 - Clear & $79(10.4)$ & $74(14.6)$ & $5(2.0)$ \\
\hline 1 - Almost Clear & $161(21.1)$ & $144(28.3)$ & $17(6.7)$ \\
\hline 2- Mild & $247(32.4)$ & $187(36.8)$ & $60(23.5)$ \\
\hline 3- Moderate & $249(32.6)$ & $93(18.3)$ & $156(61.2)$ \\
\hline 4- Severe & $23(3.0)$ & $7(1.4)$ & $16(6.3)$ \\
\hline PASI, mean (SD) ${ }^{\dagger}$ & $4.8(4.72)$ & $3.3(3.53)$ & $7.7(5.39)$ \\
\hline BSA affected, \%, mean (SD) ${ }^{\dagger}$ & $4.7(5.60)$ & $3.3(4.74)$ & $7.3(6.21)$ \\
\hline
\end{tabular}

Tapinarof $\rightarrow$ Tapinarof: patients previously assigned to tapinarof in the pivotal trials who enrolled in PSOARING 3 ,

FFour patients 3 previously assigned to tapinaroff, 1 previously yssigned to vehicle) did not have a baseline
PGA, PASI, and BSA value and are listed as missing. ITT population.

BMI, body mass index; BSA, body surface area; ITT, intention-to-tt.
Index; PGA, Physician Global Assessment, SD, standard deviation.

Complete Disease Clearance (PGA of 0)

Overall, 40.9\% (312/763) of patients achieved complete disease clearance at least

once during the study: this included 233 patients who entered the study with a

PGA of $\geq 1$, and 79 patients who entered with a PGA of 0 (Figure 2a

Response Among Patients Entering With a PGA of $\geq 2$

Overall, 58.2\% (302/519) of patients entering the study with a PGA of $\geq 2$ achieved a PGA of 0 (clear) or 1 (almost clear) at least once during the study (Figure $\mathbf{2 b}$ )

Figure 2. Complete Disease Clearance (PGA=0) and Response Rates PGA $=0$ or 1 )

a. Complete disease clearance (PGA=0)

b. Response (PGA=0 or 1 among
patients entering with a $P G A \geq 2$ )
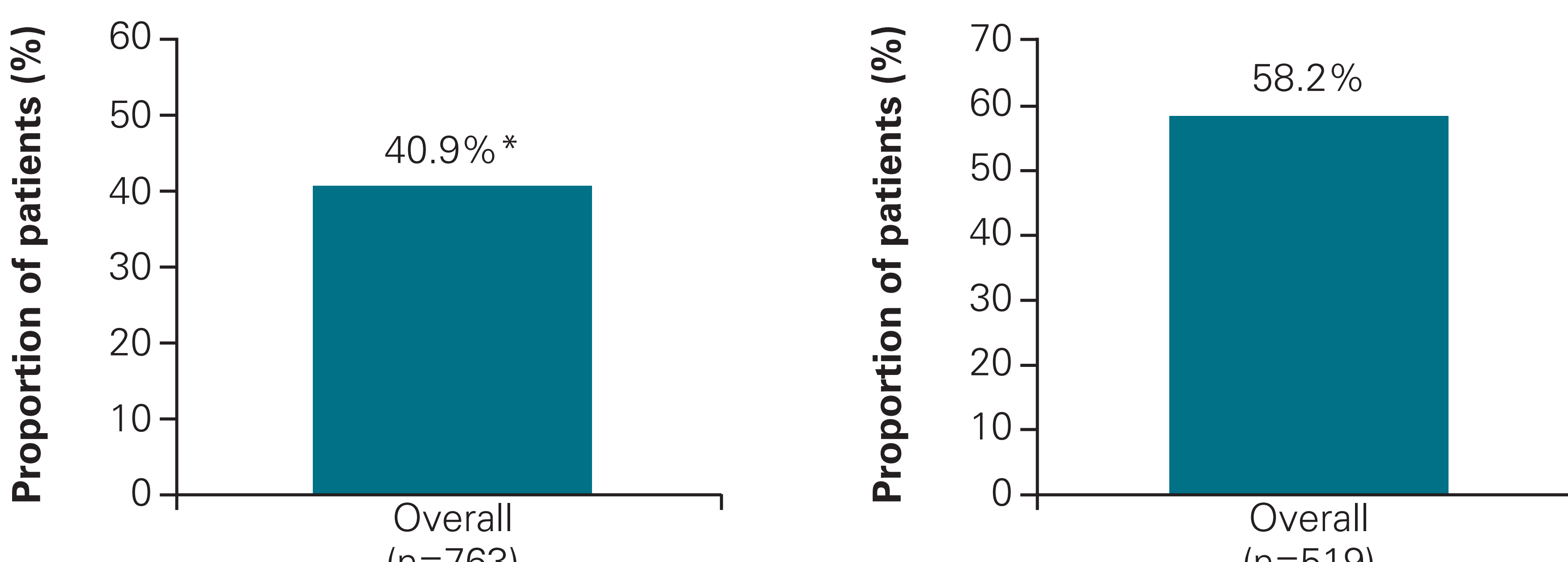

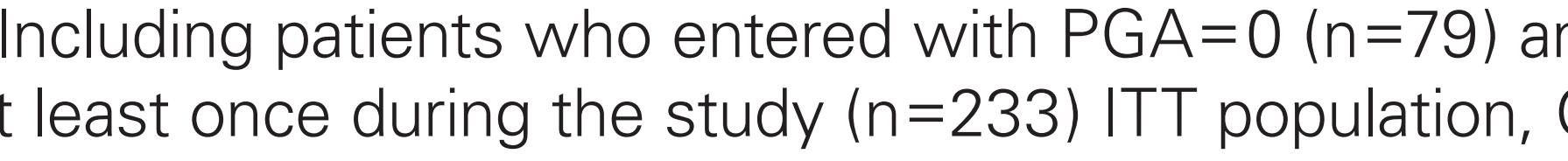

Remittive Effect: Time To First Worsening Among Patients Entering with a PGA of 0 ( $n=79)$

The duration of remittive effect (Kaplan-Meier estimated median, 95\% confidence interval [CI]) while off therapy for patients who entered the study with a PGA of 0

3. Duration of Remittive Effect Among Patients Entering With a PGA of 0: Maintenance of a PGA of $\mathbf{0}$ (Clear) or 1 (Almost Clear) While Off therapy

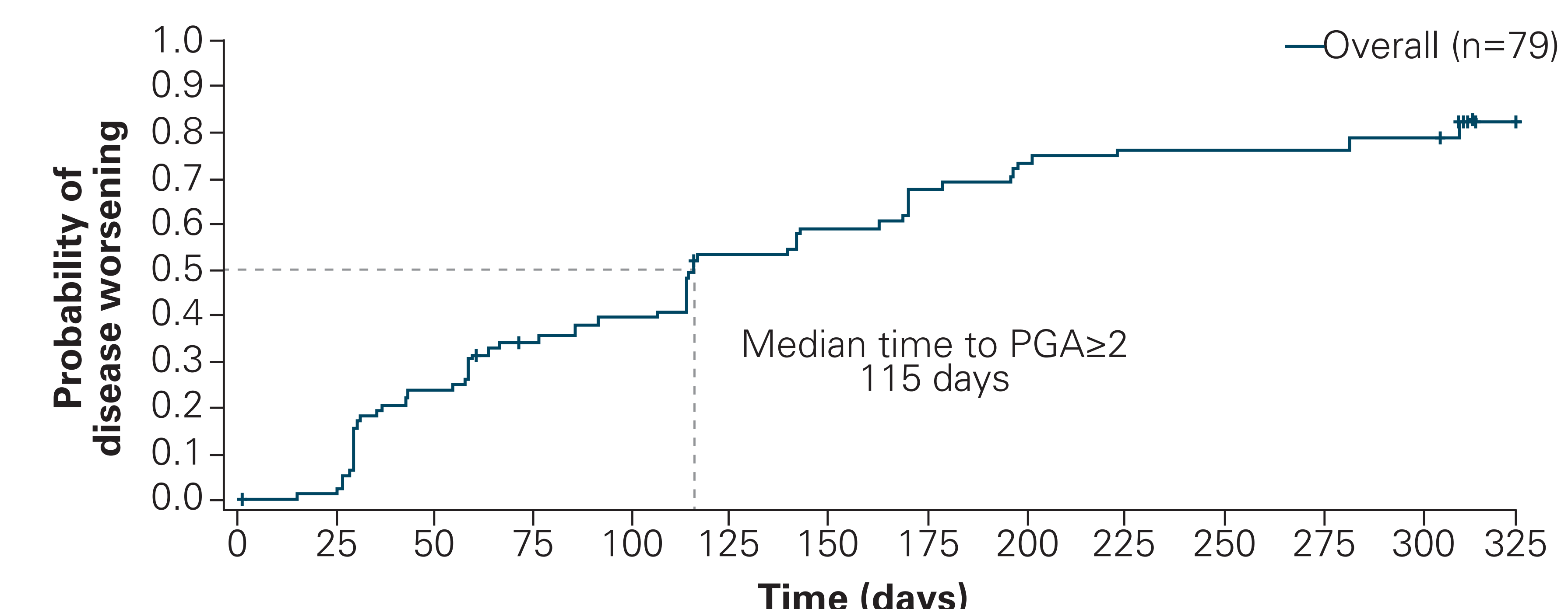

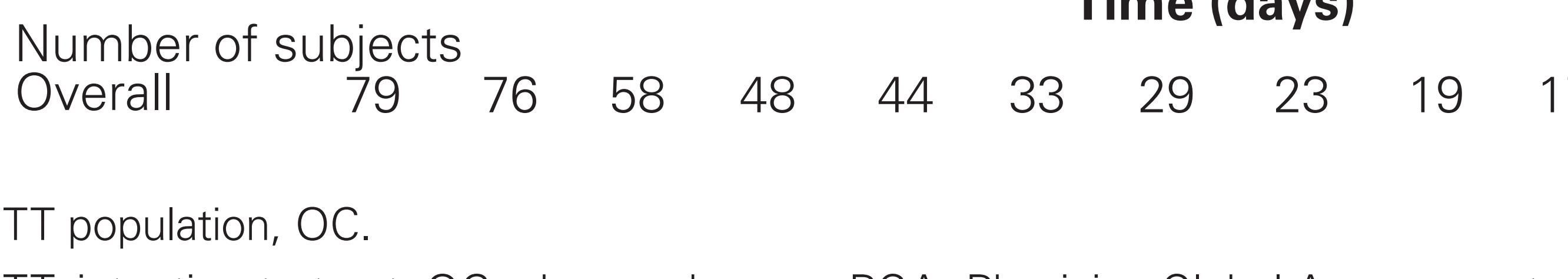

Total Duration of Remittive Effect Among Patients Entering With, or Achieving, a PGA of 0 ( $n=312$ )

The total duration of remittive effect (mean, standard deviation [SDI) while off

therapy was 130.1 (89.4) days, a possible underestimate as study end, not disease worsening, truncated the duration for some patients

Durability of Response

Durability of response of up to 52 weeks was demonstrated with intermittent use tapinarof $1 \% \mathrm{QD}$, indicating response) while on th

- Patients previously treated with vehicle in the 12-week pivotal trials achieved similar responses to patients previously treated with tapinarof (Figure 4)

Figure 4. Durability of Response (no tachyphylaxis while on therapy) Based on Proportion of Patients Achieving a PGA Score of 0 (Clear) or 1 (Almost Clear) at least Once During the Study
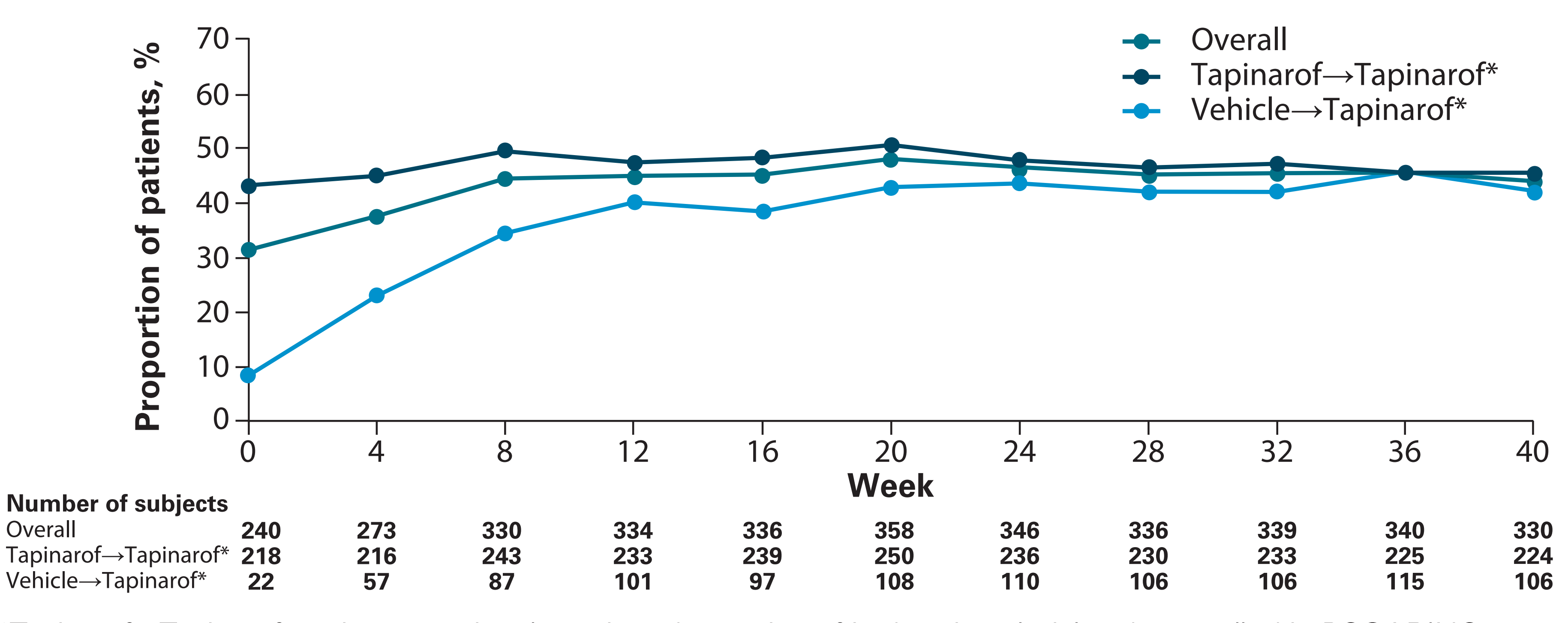

\section{Tapinarof $\rightarrow$ Tapinarof: patients previously assigned to tapinarof in the pivotal trials who enrolled in PSOARING 3 ,}

ITsessm

As previously reported, there were no new safety signals during the long-term

safety trial ${ }^{3}$ and AEs were consistent with previous studies

The most common treatment-emergent AEs included folliculitis, contact

dermatitis, and upper respiratory tract infection

Study discontinuation due to folliculitis and contact dermatitis was low, 1.2\% ( PSOARING 1 and PSOARING

\section{CONCLUSIONS}

Tapinarof cream 1\% QD provided sustained improvement in efficacy endpoints with long-term intermittent use

of complete disease clearance $(40.9 \%)$ and a remittive effect of proximately 4 months off therapy was demont

Tapinarof cream 1\% OD was well tolerated with long-term use and had a safety profile consistent with previous studies'

\section{REFERENCES}

1. Lebwohl M, et al. Skin. 2020;4(6): $: 575 ; 2$. Robbins K et al. J Am Acad Dermatol. 2019;80:714-721;
3. Strober B, et al. Innovations in Dermatology Virtual Spring Conference 2021, Poster Presentation,
March 16-20. 2021.

\section{ACKNOWLEDGMENTS}

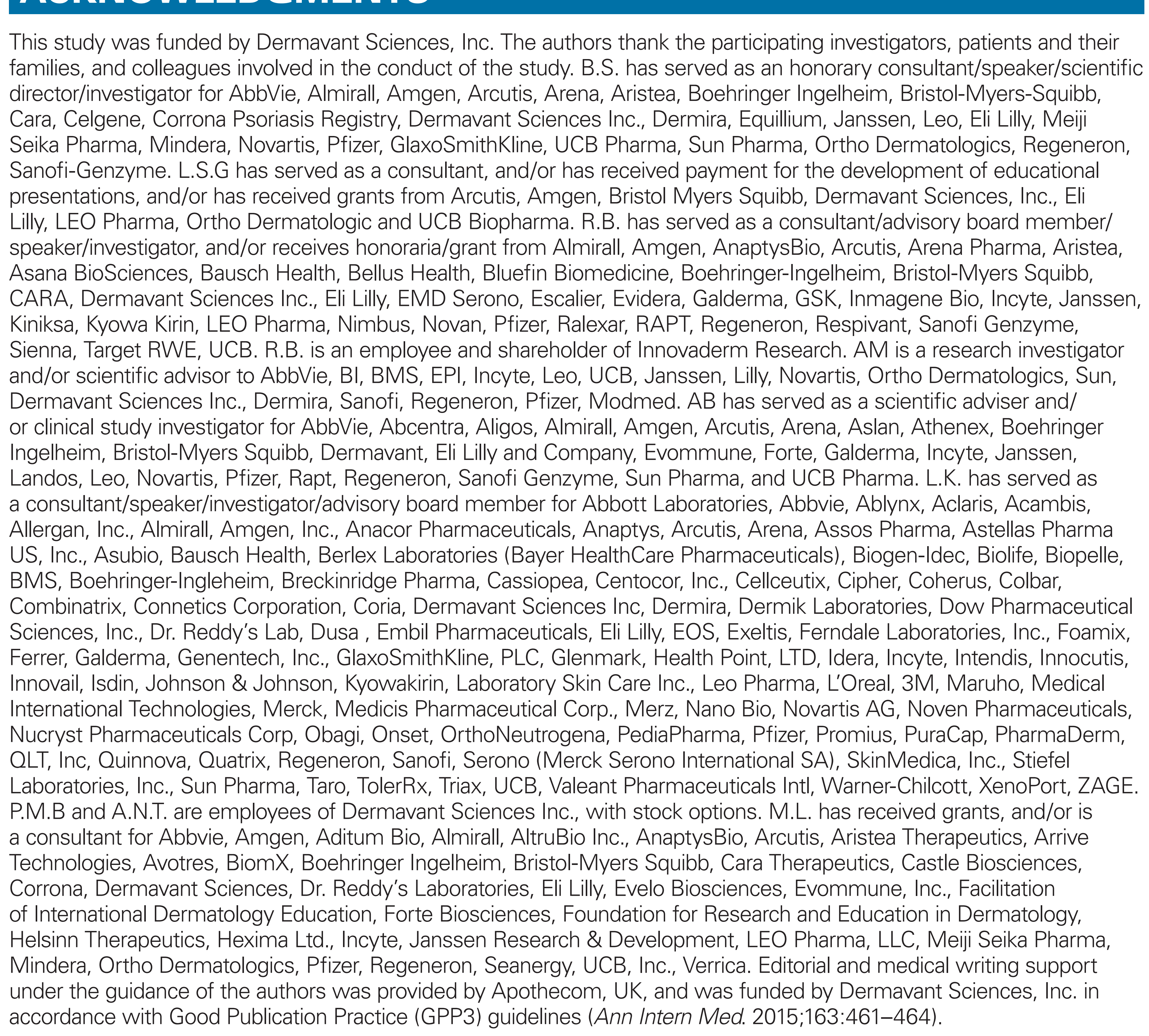

